\title{
Prediction of the Vapor-Liquid Equilibrium of Formaldehyde-Water-Trioxane Ternary System by the Conductor-Like Screening Model for Real Solvents
}

\author{
Huihong Liu, Zhenmin Bai, Yansheng Liu, Xuqiang Guo, and Yu'e Fu
}

\begin{abstract}
In this study, a thermodynamic model was combined with the conductor-like screening model for real solvents (COSMO-RS), coupled with chemical reaction equilibrium and mass balance, for describing the phase behavior and complex chemical equilibrium in the ternary system of formaldehyde-water-trioxane at temperatures between $413 \mathrm{~K}$. New approach for the prediction of vapor-liquid equilibrium of the binary system $(\mathrm{TOX}+\mathrm{W})$ and the ternary system $(\mathrm{FA}+\mathrm{W}+\mathrm{TOX})$ is compared with experimental data and UNIFAC method. The calculated vapor-liquid equilibrium (VLE) result shows good agreement with the experimental data. The COSMO-RS model has proven to be an accurate and effective method for predicting the activity coefficient, and also more reasonable for predicting the thermodynamic properties of formaldehyde-watermethanol reactive fluids.
\end{abstract}

Index Terms-COSMO-RS, modeling, phase equilibrium, formaldehyde aqueous solution, trioxane.

\section{INTRODUCTION}

Trioxane is a cyclic trimer of formaldehyde. It is an important intermediate in the production of polyacetal plastics from formaldehyde. In the production of such chemicals, trioxane with high purity is recovered by distillation from products of the formaldehyde conversion reaction in acidic aqueous solutions. The basic design of such processes requires, for example, a model for the vapor-liquid equilibrium of the chemical reactive system formaldehyde + water + trioxane [1]. A physicochemical model used in the description of vapor-liquid equilibrium in formaldehyde-containing mixtures was originally developed by Maurer [2], and has been continuously updated [1], [3]-[7].

This model have adopted UNIFAC or modified UNIFAC approaches for calculating activity coefficients. However, despite the undoubted merits of these methods, they are hindered by the requirement of time consuming experimental datasets for deriving a suitable fragmentation and the corresponding increments. Moreover, both the

Manuscript received June 20, 2016; revised August 28, 2016.

Huihong Liu is with College of Chemical Engineering, China University of Petroleum-Beijing, China. He is also with Centre of Technology \& Research, China Bluechemical Ltd., CNOOC and State Key Laboratory of Heavy Oil Processing, China University of Petroleum-Beijing, China.

Zhenmin Bai, Yansheng Liu, and Xuqiang Guo are with College of Chemical Engineering, China University of Petroleum-Beijing, and he is also with State Key Laboratory of Heavy Oil Processing, China University of Petroleum-Beijing, China.

Yu'e Fu is with Centre of Technology \& Research, China Bluechemical Ltd., CNOOC, China (e-mail: wsuper@cup.edu.cn).
UNIFAC and modified UNIFAC methods suffer from the inability to distinguish between isomers, and produce inaccuracies when compounds with strong interactions of nonalkyl functional groups are considered [8], [9]. Moreover, for aqueous formaldehyde solutions, MGs cannot be obtained as pure compounds, and precipitation is common at high formaldehyde concentrations and at low temperatures. Hence, studying thermodynamic behaviors based on experiments alone becomes difficult. Because of these challenges, it is essential to develop a novel and efficient method for predicting the thermo-physical data of liquids as an alternative to UNIFAC methods [10].

Based on the early work of Klamt, a conductor-like screening model for real solutions (COSMO-RS) [11] was proposed in 1995, and has been intensively developed over the past decades [12]-[16]. COSMO-RS is a statistical thermodynamics theory based on COSMO polarization charge densities, which overcomes many of the limitations and theoretical shortcomings of dielectric continuum models. COSMO-RS enables us to predict equilibrium in situations where measurements are not available, even for mixtures of rare, unstable, and complicated chemical species, it has become very popular in chemical engineering and in wide areas of physical and medicinal chemistry [17], [18].

Continuing previous work on the predicting of phase equilibrium in the formaldehyde-water binary system by the physicochemical model combining with COSMO-RS model [10], the method is extended to the FA+W+TOX ternary system in this study.

\section{COSMO-RS METHOD AND THERMODYNAMIC MODEL}

Formaldehyde is highly reactive and therefore forms different adducts with the solvents in aqueous solutions. The most important chemical-reaction products are methylene glycol $\left(\mathrm{HOCH}_{2} \mathrm{OH}\right.$; here, also, $\left.\mathrm{MG}\right)$ and poly (oxymethylene) glycols $\left(\mathrm{HO}\left(\mathrm{CH}_{2} \mathrm{O}\right)_{\mathrm{n}} \mathrm{H}, n>1\right.$; here, also, $\left.\mathrm{MG}_{\mathrm{n}}\right)$ as shown in Fig. 1.

Hence, in the vapor-liquid equilibrium model for FA+W+TOX mixtures, both chemical reactions and physical interactions between all species were taken into account.

As the most comprehensive studies on the physical equilibria of reactive fluids originate from the Maurer group, we adopt Maurer's description of a physiochemical model [2]. This model includes the chemical and physical equilibria of the reactive molecules. In this work, the COSMO-RS model was chosen to calculate the physical interactions of all species using their activity coefficients. 


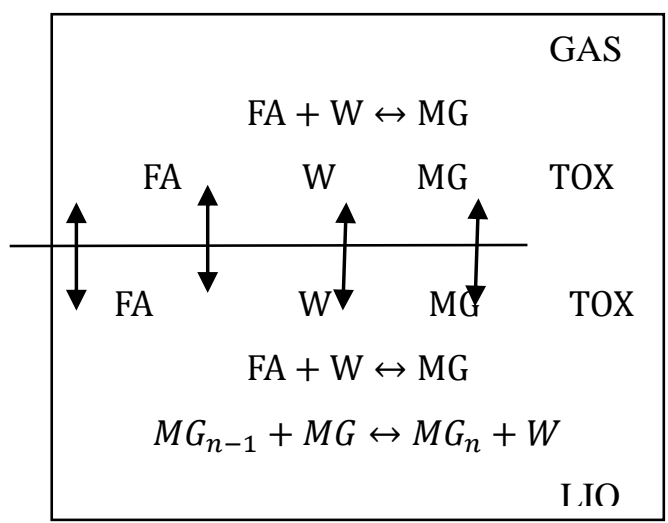

Fig. 1. Scheme for modeling the vapor-liquid equilibrium (VLE) and the chemical equilibrium for a FA+W+TOX system.

\section{A. Thermodynamic Predicted Model}

In this work, we adopt Maurer's description of chemical model [2], the detailed description of the Equilibrium Distribution Model can be found in reference [19]. This model includes the chemical and physical equilibria of the reactive molecules. In this work, the COSMO-RS model was chosen to calculate the physical interactions of all species using their activity coefficients, while the chemical phenomena were described using $K_{n}{ }^{*}$. The vapor phase is assumed to behave like a mixture of ideal gases as described in previous publications [2], [5], [7]. The criterion for phase equilibrium can be expressed according to Eq. (1), where $P_{i}^{s}, x_{i}$, and $\gamma_{i}$ are the saturated vapor pressure, true concentrations, and the corresponding activity coefficients, respectively. $P$ and $y_{i}$ represent the total pressure and composition in the gas phase.

$$
P_{i}^{s} \cdot x_{i} \cdot \gamma_{i}=P \cdot y_{i}
$$

We take chemical equilibrium constants $K_{M G}^{V}$, and vapor pressure from literatures [6], see Table I, Table II We use NMR data [20], [21] and COSMO-RS method to determine the chemical equilibrium constants $K_{M G_{n}}^{L} n \geq 2$ [10].

TABLE I: PURE COMPONENT SATURATION VAPOR PRESSURE

\begin{tabular}{|c|c|c|c|}
\hline Component & A & $\mathrm{B}$ & $\mathrm{C}$ \\
\hline Formaldehyde & 14.4625 & -2204.13 & -30.00 \\
\hline Water & 16.2886 & -3816.44 & -46.13 \\
\hline Methylene glycol & 19.5527 & -6189.19 & -9.15 \\
\hline Trioxane & 14.3796 & -3099.47 & -68.92 \\
\hline
\end{tabular}

TABLE II: CHEMICAL REACTION EQUILIBRIUM CONSTANTS $\ln K=A+B /(T / K)$

\begin{tabular}{|c|c|c|}
\hline Chemical equilibrium constant & $\mathrm{A}$ & $\mathrm{B}$ \\
\hline$K_{M G}^{V}$ & -16.984 & 5233.2 \\
\hline$K_{M G_{2}}^{L}$ & 0.00498 & 582.044 \\
\hline$K_{M G_{n}}^{L} n \geq 3$ & 0.01908 & 447.345 \\
\hline
\end{tabular}

The activity coefficient $\gamma_{i}$ is derived from the chemical potential $\mu_{i}$, which is calculated through non-linear equations in the COSMOtherm implementation (Eq. (2)).

The species concentration $x_{i}$ is calculated by combination of the thermodynamic chemical equilibrium constant $K_{n}$ * (Eq. (3)-(6)) and the appropriate mass balance equations (Eq. (7)-(9)).

$$
\begin{gathered}
\ln \gamma_{i}=\left(\mu_{i}-\mu_{0, i}\right) / R T \\
K_{M G}^{V}(T)=\left(y_{M G} /_{y_{F}} y_{W}\right) \cdot\left(P^{\theta} / P_{e q}\right) \\
K_{M G}^{L}(T)=\frac{x_{M G}}{x_{F A} x_{W}} \cdot \frac{\gamma_{M G}}{\gamma_{F A} \gamma_{W}}=K_{M G}^{V}(T) \frac{P_{F A}^{S} P_{W}^{S}}{P_{M G}^{S} P^{\theta}} \\
K_{M G_{n}}^{L}=\frac{x_{M G_{n}} x_{W}}{x_{M G_{n-1}} x_{M G}} \cdot \frac{\gamma_{M G_{n}} \gamma_{W}}{\gamma_{M G_{n-1}} \gamma_{M G}} n \geq 2 \\
K_{M G_{2}}^{L} \neq K_{M G_{3}}^{L}=K_{M G_{4}}^{L}=\cdots=K_{M G_{n}}^{L}=\cdots \\
\tilde{x}_{F A}=S \cdot\left(x_{F A}+x_{M G}+\sum_{n=2}^{8} n \cdot x_{M G_{2}}\right) \\
\tilde{x}_{w}=S \cdot\left(x_{w}+x_{M G}+\sum_{n=2}^{8} x_{M G_{n}}\right) \\
\mathrm{S}=\left(1+x_{M G}+\sum_{2}^{8} n \cdot x_{M G_{n}}\right)^{-1}
\end{gathered}
$$

\section{B. COSMO-RS Method}

A detailed description of the COSMO-RS method is beyond the scope of this article but has been described in the literature [15]-[18]. Thus, only the basic features required for understanding the results of our study shall be described.
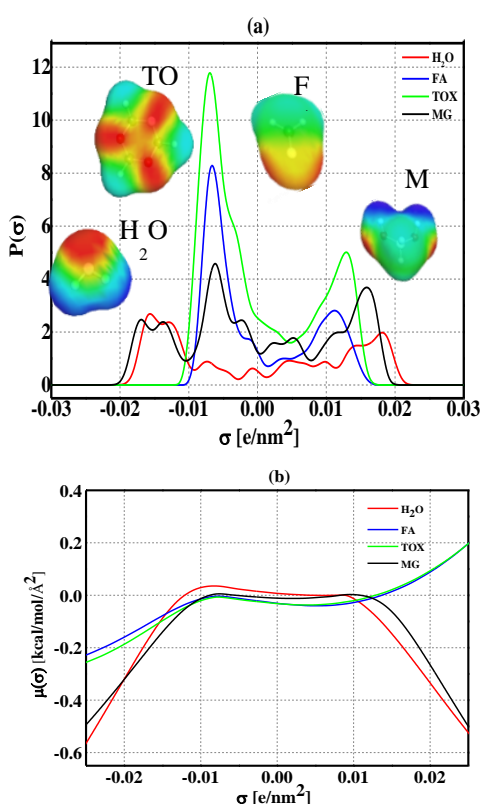

Fig. 2. Illustration of the $\sigma$-profiles (a) and $\sigma$-potential (b) of formaldehyde, water, MG and TOX.

This study involves 9 molecules, namely, FA, W, TOX, $\mathrm{MG}_{n}(6 \geq n \geq 1)$. Water, TOX, and FA molecules were included in the COSMOtherm database without any geometry optimization. TmoleX software was utilized for the building the structures of $\mathrm{MG}_{n}(6 \geq n \geq 1)$, as well as the optimization of geometries at the HartreeFocklevelanddef-TZVP basis set for each molecule [22]. Based on Cosmo files, the probability distribution of surface segments, referred to as the " $\sigma$-profile," as well as " $\sigma$-potential," which indicates the affinity between solvent and solute molecules, was obtained. In addition, the activity 
coefficient of molecules involved was determined. In this study, all of the above COSMO-RS calculations were performed using the COSMOtherm program12.01 [23].

The 3D polarization density distribution on the surface of molecule, the $\sigma$-profile and $\sigma$-potential of FA, W, MG, and TOX are shown in Fig. 2 (a) and (b).

\section{Combination of the Chemical Reactions with COSMO-RS Software Using MATLAB Codes}

As we know, the description of chemical equilibrium conditions in the thermodynamic equilibrium constants $\mathrm{K}_{n}{ }^{*}$ introduced the iterative calculation of composition $x_{i}$ and activity coefficients $\gamma_{i}$ in formaldehyde aqueous solution. In other words, it is necessary to combine the chemical reactions with the COSMO-RS software, and this combination can be solved using the function reference in the MATLAB program.

Our method links the activity coefficient modules in COSMOtherm with the chemical reaction equilibrium constants and mass balance equations with the aid of the MATLAB program, which contains powerful reference functions. The fopen function in MATLAB was employed to run the "inp" profile and to give the order to run the activity coefficients module. To ensure that the coefficients are assigned to the chemical reaction equilibrium constants (Eq. (3)-(6)) and mass balance equations (Eq. (7)-(9)), the species distribution values were obtained. Calculation of each piece of experimental data required approximately $5 \mathrm{~s}$ of computational time.

\section{COMPARISON WITH EXPERIMENTAL RESULTS}

To check the validity of this approach to predict VLE of FA+W+TOX ternary system, the VLE and species distribution of the FA-W reactive binary system have been precisely predicted by the COSMO-RS method in our previous study, the average deviations of vapor-phase composition and pressure are relatively $1.75 \%$ and $1.49 \%$ [10]. Further, the predicted VLE result of TOX $+\mathrm{W}$ binary system and FA+W+TOX ternary system were compared to the experiment data and calculated data using UNIFAC model taken from the literature [1].

\section{A. Prediction of the TOX-Water Binary System}

The partition coefficient of trioxane and vapor pressure of TOX $+\mathrm{W}$ binary system at $413 \mathrm{~K}$ is shown in Fig. 3 (a) and (b). Also, Fig. 3 shows the comparison of the correlation between our model and previous UNIFAC model in literature [1]. In comparison to the experimental data reported by Maurer, the average relative deviations are $2.7 \%$ and $0.53 \%$ for the partition coefficient of trioxane and the pressure, respectively. The agreement between the correlation results and the experimental data is considered to be satisfactory. Typically, the partition coefficient of trioxane and formaldehyde calculated by the revised model in the work is closer to the experimental data comparing with the model in literatures from Fig. 3.

The comparison between the results of the correlation and the experimental data in literatures is given in Table III The standard deviations between the calculated data of our work and reported by Albert (16 data points at $413 \mathrm{~K}$ ) for the vapor-phase concentrations of $\mathrm{f}$ trioxane and the pressure are smaller, respectively.
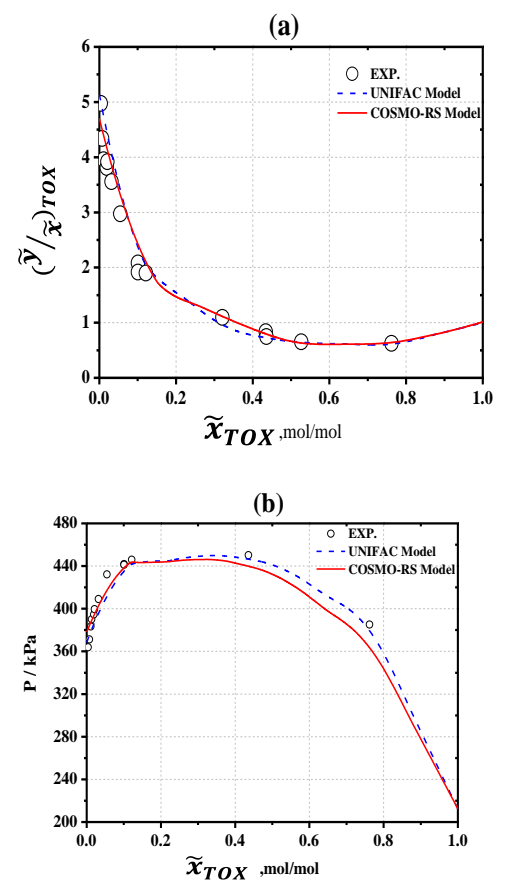

Fig. 3. Participation coefficient (a) and Vapor pressure (b) above aqueous solutions of (trioxane+ water) at $413 \mathrm{~K}$ : Comparison between the experimental results [1], the correlation of the UNIFAC [1] and present work.

TABLE III: DIVISION OF Correlation RESULTS (COSMO-Rs (1) AND UNIFAC (2) [1]) FOR THE VAPOR-LIQUID EQUILIBRIUM OF THE TRIOXANE + WATER BINARY SYSTEM

\begin{tabular}{|c|c|c|c|c|c|c|}
\hline \multirow{2}{*}{$T(K)$} & \multirow{2}{*}{$X_{\mathrm{w}}^{\sim}$} & \multirow{2}{*}{$\tilde{x}_{T O X}$} & \multicolumn{2}{|c|}{$\tilde{y}_{F A}$} & \multicolumn{2}{|c|}{$P$} \\
\hline & & & $\Delta y^{\sim} 1 / \%$ & $\Delta y \sim 2 / \%$ & $\Delta p_{I} / \%$ & $\Delta p_{2} / \%$ \\
\hline 412.2 & 0.996 & 0.004 & 0.0676 & 0.0753 & -0.0067 & -0.0164 \\
\hline 412.5 & 0.9929 & 0.0071 & 0.1252 & 0.1915 & -0.0001 & -0.0153 \\
\hline 413.2 & 0.989 & 0.011 & 0.1095 & 0.2367 & 0.0067 & -0.0125 \\
\hline 413.2 & 0.9868 & 0.0132 & -- & -- & -0.0004 & -0.0210 \\
\hline 413.1 & 0.9808 & 0.0192 & 0.0810 & 0.1666 & 0.0083 & -0.0179 \\
\hline 413.2 & 0.9789 & 0.0211 & 0.0091 & 0.1124 & 0.0048 & -0.0212 \\
\hline 413.1 & 0.9681 & 0.0319 & 0.0444 & 0.0970 & 0.0095 & -0.0198 \\
\hline 413.2 & 0.9454 & 0.0546 & 0.0082 & 0.0665 & -0.0079 & -0.0300 \\
\hline 413.1 & 0.8993 & 0.1007 & 0.0042 & 0.0891 & -0.0043 & -0.0126 \\
\hline 413.2 & 0.8991 & 0.1009 & 0.0690 & 0.1826 & 0.0001 & 0.01541 \\
\hline 413 & 0.8788 & 0.1212 & 0.0003 & 0.0560 & -0.0107 & -0.0134 \\
\hline 413.2 & 0.679 & 0.321 & -0.0304 & -0.1598 & -- & -- \\
\hline 413.2 & 0.5658 & 0.4342 & 0.0689 & -0.1355 & -- & -- \\
\hline 413 & 0.564 & 0.436 & -0.0894 & -0.0382 & -0.0249 & -0.0099 \\
\hline 413.1 & 0.4733 & 0.5267 & -0.0470 & -0.0258 & -- & -- \\
\hline 413.1 & 0.2381 & 0.7619 & -0.0154 & -0.0515 & -0.0441 & 0.0005 \\
\hline \multicolumn{3}{|c|}{ Average relative deviation } & 0.0270 & 0.0575 & 0.0053 & 0.0134 \\
\hline
\end{tabular}

\section{B. Prediction of FA+W+TOX Ternary System}

As mention above, our model gives a good representation of vapor-liquid equilibrium data in binary systems of $\mathrm{FA}+\mathrm{W}$ and $\mathrm{TOX}+\mathrm{W}$ over the entire composition ranges where reliable experimental data is available. Deviations between measured and calculated values are typically below $5 \%$ for the partition coefficient of formaldehyde and $2 \%$ for the pressure. In the extension of that model to the ternary 
system $(\mathrm{FA}+\mathrm{W}+\mathrm{TOX})$, and trioxane is considered to be an inert substance of formaldehyde-water binary system.

Table IV reports a comparison between experimental data in the literature [1], and Table $\mathrm{V}$ shows the comparison of the average overall deviation of the gas-phase composition and pressure for the ternary system. As can be observed from the deviation table, compared to the UNIFUAC model, our COSMO-RS model provides a more accurate description.

TABLE IV: EXPERIMENTAL RESULTS [1] AND CORRELATION RESULTS (COSMO-RS (1) AND UNIFAC (2)[1]) FOR THE VAPOR-LIQUID EQUILIBRIUM OF THE FA+W+TOX TERNARY SYSTEM

\begin{tabular}{|c|c|c|c|c|c|c|c|c|c|c|c|}
\hline \multirow{2}{*}{$\mathrm{T}(\mathrm{K})$} & \multirow{2}{*}{$\tilde{x}_{F A}$} & \multirow{2}{*}{$\tilde{\mathrm{x}}_{\mathrm{TOX}}$} & \multicolumn{3}{|c|}{$\tilde{y}_{F A}$} & \multicolumn{3}{|c|}{$\tilde{y}_{\text {TOX }}$} & \multicolumn{3}{|c|}{$\mathrm{P}$} \\
\hline & & & Exp & Cal1 & Cal2 & Exp & Cal1 & $\mathrm{Cal} 2$ & Exp & Cal1 & $\mathrm{Ca} 2$ \\
\hline 413.1 & 0.109 & 0.011 & 0.1865 & 0.1995 & 0.1967 & 0.0378 & 0.0483 & 0.0413 & 427.9 & 435.83 & 425.2 \\
\hline 413.2 & 0.105 & 0.0478 & 0.1711 & 0.1727 & 0.1736 & 0.1364 & 0.1349 & 0.1311 & 462.9 & 464.35 & 455.7 \\
\hline 413.2 & 0.136 & 0.0587 & 0.1994 & 0.1982 & 0.1996 & 0.1466 & 0.1415 & 0.1421 & 478.8 & 474.03 & 466.9 \\
\hline 413.1 & 0.04 & 0.0925 & 0.0789 & 0.0825 & 0.0821 & 0.1953 & 0.1981 & 0.2041 & 461 & 459.87 & 453.6 \\
\hline 413.1 & 0.060 & 0.0933 & 0.107 & 0.1117 & 0.1119 & 0.2013 & 0.1911 & 0.1994 & 470.1 & 467.10 & 461.9 \\
\hline 413.1 & 0.085 & 0.0953 & 0.1385 & 0.140 & 0.1403 & 0.1939 & 0.1850 & 0.1952 & 478.9 & 473.79 & 469.5 \\
\hline 413.1 & 0.046 & 0.2084 & 0.0856 & 0.0909 & 0.0906 & 0.2509 & 0.2318 & 0.2603 & 478.8 & 470.74 & 472.3 \\
\hline 413.1 & 0.024 & 0.3206 & 0.0563 & 0.0596 & 0.0607 & 0.2812 & 0.2592 & 0.2878 & 471.9 & 461.97 & 465.6 \\
\hline 413.2 & 0.035 & 0.4691 & 0.0943 & 0.0956 & 0.0889 & 0.3196 & 0.2923 & 0.3078 & 474 & 461.72 & 468.8 \\
\hline 413.2 & 0.030 & 0.528 & 0.0901 & 0.0942 & 0.0865 & 0.303 & 0.3121 & 0.3217 & 465 & 453.62 & 463.4 \\
\hline 413.2 & 0.030 & 0.6541 & 0.0936 & 0.0909 & 0.1034 & 0.3766 & 0.3643 & 0.3619 & 462.2 & 433.06 & 446.8 \\
\hline 413.3 & 0.022 & 0.8975 & 0.1673 & 0.1627 & 0.1693 & 0.5711 & 0.5635 & 0.5692 & 364.5 & 347.16 & 345.4 \\
\hline
\end{tabular}

TABLE V: DIVISION OF CORRELATION RESULTS (COSMO-RS (1) AND UNIFAC (2) [1]) FOR THE VAPOR-LIQUID EQUILIBRIUM OF THE FA+W+TOX TERNARY

\begin{tabular}{|c|c|c|c|c|c|c|c|c|}
\hline \multirow{2}{*}{$T(K)$} & \multirow{2}{*}{$\tilde{x}_{F A}$} & \multirow{2}{*}{$\tilde{x}_{T o X}$} & $\tilde{y}_{F A}$ & \multicolumn{3}{|c|}{ SYSTEM } & \multicolumn{2}{|c|}{$\boldsymbol{P}$} \\
\hline & & & $\Delta y^{\sim} 1 / \%$ & $\Delta y^{\sim} 2 / \%$ & $\Delta y^{\sim} 1 / \%$ & $\Delta y^{\sim} 2 / \%$ & $\Delta p_{1} / \%$ & $\Delta p_{2} / \%$ \\
\hline 413.1 & 0.109 & 0.011 & 0.0700 & 0.054692 & 0.02802 & 0.0925 & 0.018551 & -0.00631 \\
\hline 413.2 & 0.105 & 0.0478 & 0.0098 & 0.014611 & -0.01068 & -0.0388 & 0.003154 & -0.01555 \\
\hline 413.2 & 0.136 & 0.0587 & -0.0056 & 0.001003 & -0.03442 & -0.0307 & -0.0099 & -0.02485 \\
\hline 413.1 & 0.04 & 0.0925 & 0.0463 & 0.040558 & 0.014656 & 0.0450 & -0.00244 & -0.01605 \\
\hline 413.1 & 0.060 & 0.0933 & 0.0448 & 0.045794 & -0.05035 & -0.0094 & -0.00638 & -0.01744 \\
\hline 413.1 & 0.085 & 0.0953 & 0.0130 & 0.012996 & -0.04549 & 0.0067 & -0.01066 & -0.01963 \\
\hline 413.1 & 0.046 & 0.2084 & 0.0619 & 0.058411 & -0.07597 & 0.0374 & -0.01682 & -0.01358 \\
\hline 413.1 & 0.024 & 0.3206 & 0.0591 & 0.078153 & -0.078 & 0.0234 & -0.02103 & -0.01335 \\
\hline 413.2 & 0.035 & 0.4691 & 0.0145 & -0.05726 & -0.08532 & -0.036 & -0.02591 & -0.01097 \\
\hline 413.2 & 0.030 & 0.528 & 0.0461 & -0.03996 & 0.030173 & 0.0616 & -0.02447 & -0.00344 \\
\hline 413.2 & 0.030 & 0.6541 & -0.028 & 0.104701 & -0.03264 & -0.039 & -0.06303 & -0.03332 \\
\hline 413.3 & 0.022 & 0.8975 & -0.027 & 0.011955 & -0.0133 & -0.0033 & -0.04757 & -0.0524 \\
\hline \multicolumn{3}{|c|}{ Average relative deviationn } & 0.0253 & 0.0271 & -0.00843 & 0.0091 & -0.01721 & -0.0180 \\
\hline
\end{tabular}

\section{CONCLUSION}

Quantum chemical calculations for polymeric glycols produced by a series of reactions in aqueous formaldehyde solutions were modified based on a previously reported model, along with the chemical equilibrium constants $\mathrm{Kn} *$ for the formation of poly(oxymethylene) glycols (MGn $n \geq 1$ ). Combined with the chemical reactive equilibria and mass balance equations, the COSMO-RS model was applied to predict the vapor-liquid equilibrium (VLE) of the formaldehyde-water-trioxane system. The agreement between the experimental and predicted results was very good. Moreover, comparison of the results showed that the quantum chemical calculation model was more accurate than the previously reported UNIFAC method. Furthermore, our present COSMO-RS model can be applied to other multicomponent systems, and can be used to guide the rational design of trioxane and dioxolane synthesis and the separation processes for formaldehyde-containing mixtures.

\section{ACKNOWLEDGMENT}

The authors gratefully acknowledge financial support from the National Natural Science Foundation of China (21176248).

\section{REFERENCES}

[1] M. Albert, H. Hasse, C. Kuhnert, and G. Maurer, "New experimental results for the vapor-liquid equilibrium of the binary system (trioxane+ water) and the ternary system (formaldehyde+ trioxane+ water)," Journal of Chemical \& Engineering Data, vol. 50, no. 4, pp. 1218-1223, 2005.

[2] G. Maurer, "Vapor - liquid equilibrium of formaldehyde - and water - containing multicomponent mixtures," AIChE Journal, vol. 32, no. 6, pp. 932-948, 1986. 
[3] H. Hasse, I. Hahnenstein, and G. Maurer, "Revised vapor-liquid equilibrium model for multicomponent formaldehyde mixtures,' AIChE Journal, vol. 36, no. 12, pp. 1807-1814, 1990.

[4] H. Hasse and G. Maurer, "Vapor-liquid equilibrium of formaldehyde-containing mixtures at temperatures below $320 \mathrm{~K}$," Fluid Phase Equilibria, vol. 64, pp. 185-199, 1991.

[5] M. Albert, I. Hahnenstein, H. Hasse, and G. Maurer, "Vapor-liquid equilibrium of formaldehyde mixtures: new data and model revision," AIChE Journal, vol. 42, no. 6, pp. 1741-1752, 1996.

[6] M. Albert, B. C. Garcia, C. Kreiter, and G. Maurer, "Vapor-liquid and chemical equilibria of formaldehyde-water mixtures," AIChE Journal, vol. 45 , no. 9 , pp. 2024-2033, 1999

[7] M. Albert, B. C. Garcia, C. Kuhnert, R. Peschla, and G. Maurer, "Vapor-liquid equilibrium of aqueous solutions of formaldehyde and methanol," American Institute of Chemical Engineers. AIChE Journal, vol. 46, no. 8, p. $1676,2000$.

[8] S. T. Lin and S. I. Sandler, "Multipole corrections to account for structure and proximity effects in group contribution methods: Octanol-water partition coefficients," J. Phys. Chem, vol. 104, pp. 7099-7105, 2000.

[9] S.T. Lin and S. I. Sandler, "A priori phase equilibrium prediction from segment contribution solvation model," Ind. Eng. Chem, Res, vol. 41, pp. 899-913, 2002.

[10] Z. Bai, H. Liu, Y. Liu, and L. Wu, "Prediction of the vapor-liquid equilibrium of chemical reactive systems containing formaldehyde using the COSMO-RS method," Fluid Phase Equilib, vol. 415, pp. 125-133, 2016.

[11] M. Albert, B. C. Garcia, C. Kuhnert, C. Kuhnert, R. Peschla, and G. Maure, "Vapor-liquid equilibrium of aqueous solutions of formaldehyde and methanol," AIChE, vol. 46, pp. 1676-1687, 2000.

[12] M. Decheberry, P. Destrac, X.-M. Meyer and J.-S. Condoret, "Phase equilibria of aqueous solutions of formaldehyde and methanol: Improved approach using UNIQUAC coupled to chemical equilibria,' Fluid Phase Equilib. vol. 392, pp. 84-94, 2015.

[13] S. T. Lin and S. I. Sandler, "Multipole corrections to account for structure and proximity effects in group contribution methods: Octanol-water partition coefficients," J. Phys. Chem, vol. 104, pp. 7099-7105, 2000.

[14] S. T. Lin and S.I. Sandler, "A priori phase equilibrium prediction from segment contribution solvation model," Ind. Eng. Chem, Res., vol. 41, pp. 899-913, 2002.

[15] A. Klamt, "Conductor-like screening model for real solvent: A new approach to the quantitative calculation of salvation phenomena," $J$. Phys. Chem, vol. 99, pp. 2224-2235, 1995.

[16] A. Klamt, V. Jonas, T. Burger and J.-C.-W. Lohrenz, "Refinement and parameterization of COSMO-RS," J. Phys. Chem, A., vol. 102, pp. 5074-5085, 1998.
[17] A. Klamt, G. Krooshof, and R. Taylor, "COSMOSPACE: "Alternative to conventional activity coefficient models," AIChE Journal, vol. 48, pp. 2332-2349, 2002.

[18] A. Klamt, "The COSMO and COSMO-RSsolvation models," WIREs Computational Molecular Science, vol. 1, pp. 699-709, 2011.

[19] G. Li, C. Shi and Y. H. Y. Liu, "Vapor-liquid equilibrium of trioxane-formaldehyde-water mixtures - New division of components in Unifac groups," Aiche Spring National Meeting, 2013.

[20] I. Hahnenstein, H. Hasse, Y. Q. Liu, and G. Maurer, "Thermodynamic properties of formaldehyde containing mixture for separation process design," AIChESymp. Ser, vol. 90, pp. 141, 1994

[21] I. Hahnenstein, M. Albert, H. Hasse, C. G. Kreiter, and G. Maurer, "NMR-Spectroscopic and densimetric study of reaction kinetics of formaldehyde polymer formation in water, deurerium oxide and methanol," Ind. Eng. Chem. Res, vol. 34, p. 440, 1995.

[22] Turbomole, Version 6.20, TURBOMOLE GmbH, Germany, 2010.

[23] COSMOtherm Program, Version C30_1201, COSMOlogic, GmbH\&CoKG, Leverkusen, Germany, 2012.

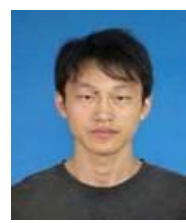

Huihong Liu was born in Huocheng Xinjiang, P. R. China on December $26^{\text {th }}, 1984$. He received his Ph.D. of chemical technology from Chemical Engineering Institute, China University of Petroleum, Beijing, China in August 2012. The dissertation title is "Prediction of thermodynamic properties of chemical reactive systems containing formaldehyde via COSMO-RS method." He got the master of chemical technology degree in Chemical Engineering Institute, Hainan University, Haikou, China in June 2010. He received his bachelor of chemical technology degree in the same university in June 2007.

He worked in Centre of Technology \& Research, China Bluechemical Ltd., CNOOC, Dongfang, China during Aug. 2010-Aug. 2012 as Researching Engineer, who is majoring in process optimization of 3000 tons/year PPC (Poly (propylene carbonate)) Plant. He worked in Centre of Technology \& Research, China Bluechemical Ltd., CNOOC, Hohhot, China during Aug. 2012 -Jun. 2015 as Researching Engineer majoring in process optimization of 60,000 tons/year POM (polyoxymethylene) Plant. He worked in Centre of Technology \& Research, China Bluechemical Ltd., CNOOC, Beijing, China during Aug. 2013 -Jun. 2014 majoring in management of scientific research projects. $\mathrm{He}$ is working in Centre of Technology \& Research, China Bluechemical Ltd. CNOOC, Dongfang, China. Since Jun. 2015 as Researching Engineer majoring in Quantum chemical calculation in the application of the chemical process.

Dr. Liu was awarded 'First-class scientific and technological progress award' by China Bluechemical Ltd., CNOOC in 2014 and by Hainan province in 2016. 